\title{
Design of Controller for Large Scale Uncertain Systems via Reduces Order Model
}

\author{
Vudikala Lalitha ${ }^{1} \mid$ Dr. T Narasimhulu ${ }^{1}$ \\ ${ }^{1}$ Department of EEE, ANITS.
}

To Cite this Article

Vudikala Lalitha and Dr. T Narasimhulu, "Design of Controller for Large Scale Uncertain Systems via Reduces Order Model”, International Journal for Modern Trends in Science and Technology, 6(12): 262-267, 2020.

\section{Article Info}

Received on 10-November-2020, Revised on 02-December-2020, Accepted on 06-December-2020, Published on 11-December-2020.

\section{ABSTRACT}

In This paper, a method of designing the Controller for large scale uncertain systems. The Controller is designed via a reduced order model for a given high order system. An optimized reduced order model is derived with minimum ISE. The proposed method guarantees stability of the reduced model, if the original high order system is stable system. A PID controller is designed for the high order original systems through its low order model proposed. This paper presents an improvement to generalized least squares method of model order reduction. The improvement enhances the flexibility of the method with very little computational requirement. The reduction procedure is simple, efficient and always generates stable reduced models for the stable high order systems. The proposed method is illustrated with typical numerical examples taken from the literature and the results are compared with the other existing methods to show its superiority.

KEYWORDS:Improved least squares method, Integral Square Error, Order reduction, Moment Matching.

\section{INTRODUCTION}

The mathematical description of most physical systems leads to higher order differential equations which are difficult to use either for analysis or controller synthesis. It is hence useful and sometimes necessary to find the possibility of finding some equation of the same type but of lower order that may be considered to adequately reflect the dominant characteristics of the system under consideration. Numerous methods are available in the literature for order reduction of linear continuous systems in time domain as well as in frequency domain [1]-[3]. Basing on the simplicity and amicability the frequency domain dependent methods have become more prominent. Transfer function reduction methods are one of the important groups in the frequency domain category.

A popular approach, known as Pade approximation method[4]-[5] for deriving reduced order models has been based on matching of the time moments of original and reduced order Systems. A serious drawback of this method is generating unstable reduced model for a stable higher order model. To overcome this problem Shoji et al[6] suggests using a least squares time moment to obtain a reduced transfer function denominator, and numerator by exact time moment matching. This method has been refined by Lucas and Beat[7], in which the linear shift point was about general point ' $a$ ', where $a \approx(1-a)$ and $-a$ is the real part of the smallest magnitude pole. Further the method of model order reduction by least squares moment matching was generalized [8] by including the Markova parameters in the process to cope with a wider class of transfer function. On the other hand, Aguirre [9] has argued that one of the chief advantages of the least squares Pade (LS-Pade) method is that additional information concerning the original system over 
the mid-frequency range is included in the simplified model, and consequently better approximations are often obtained. Recently Parmar and Prasad et al[10] entended the concept of order reduction by least squares moment matching and generalized least squares methods about general point ' $a$ ' in order to have better approximations, Some heuristic criteria was employed for selecting the linear shift point ' $a$ ', based upon the means (arithmetic, harmonic and geometric) of real parts of the poles of higher order system. In spite of the significant number of methods available, no approach always gives the best results for all systems. Almost all methods, however aim at accurate reduced models for a low computation cost. In addition, it is desired to preserve the stability of the original model; i.e., given a stable high order model, the reduced order model should also be stable.

To overcome these difficulties in this paper, the concept of order reduction by generalized least squares method has been improved in order to have better approximations of high order linear time-invariant dynamic systems for a low computational cost. The relative mapping errors between the original and reduced models are determined, time and frequency responses are plotted to show the effectiveness of the method.

\section{Methodology}

Let the transfer function of the original high order linear dynamic SISO system of order ' $\mathrm{n}$ ' be:

$G_{n}(s)=\frac{N(s)}{D(s)}=\frac{b_{0}+b_{1} s+\cdots+b_{n-1} s^{n-1}}{a_{0}+a_{1} s+\cdots+a_{n-1} s^{n-1}+a_{n} s^{n}}$

And Let the corresponding $\mathrm{r}^{\text {th }}$ order reduced model is synthesized as:

$G_{r}(s)=\frac{N_{r}(s)}{D_{r}(s)}=\frac{d_{0}+d_{1} s+\cdots+d_{r-1} s^{r-1}}{e_{0}+e_{1} s+\cdots+e_{r-1} s^{r-1}+s^{r}}$

Further, the method consists of the following steps.

Step 1: Determination of the denominator coefficients of reduced order model using generalised least squares method, Expand $G_{n}(s)$ about $\mathrm{s}=0$, to obtain the time moment proportional $\left(C_{i}\right)$ are given by

$G_{n}(s)=C_{0}+C_{1} s+C_{2} S^{2}+\cdots \cdots$

$$
=\sum_{i=0}^{\infty} C_{i} s^{i}
$$

Similarly Expand $G_{n}(s)$ about $s=\infty$, to obtain the markov parameters $\left(M_{j}\right)$ are given by:

$$
\begin{aligned}
G_{n}(s) & =M_{1} S^{-1}+M_{2} S^{-2}+M_{3} S^{-3}+\cdots \\
& =\sum_{i=1}^{\infty} \frac{M_{j}}{s^{j}}
\end{aligned}
$$

Evaluating Eq.(2) and(3) to retain ' $t$ ' Time moments of the original model gives the following set of equations:

$$
\left.\begin{array}{l}
d_{0}=e_{0} c_{0} \\
d_{1}=e_{1} c_{0}+e_{0} c_{1} \\
\vdots \quad \vdots \quad \vdots \\
d_{r-1}=e_{r-1} c_{0}+\cdots \cdots+e_{0} c_{r-1}
\end{array}\right\}
$$

Evaluating Eq.(2) and(4) to retain ' $\mathrm{m}$ ' Markov parameters of the original model gives the following set of equations:

$$
\begin{gathered}
d_{r-1}=M_{1} \\
d_{r-2}=M_{1} e_{r-1}+M_{2} \\
\vdots \quad \vdots \quad \vdots \quad \\
\left.\begin{array}{c}
M_{1} e_{0}+M_{2} e_{1}+\cdots+M_{r} e_{r-1}=-M_{r+1} \\
M_{m-r} e_{0}+\cdots+M_{m-1} e_{r-1}= \\
M_{m}
\end{array}\right)
\end{gathered}
$$

Step 2: Elimination of $d_{j}(\mathrm{j}=0,1, \ldots \ldots . \mathrm{r}-1)$ in Eq.(4) by substituting Eq.(6) gives the reduced denominator coefficients as the solution of:

$$
\left[\begin{array}{ccccc}
c_{t-1} & c_{t-2} & \cdots & c_{t-\gamma+1} & c_{t-\gamma} \\
c_{t-2} & c_{t-3} & \cdots & \cdots & c_{t-\gamma-1} \\
\vdots & \vdots & \vdots & \vdots & \vdots \\
c_{\gamma-1} & c_{\gamma-2} & \cdots & c_{1} & c_{0} \\
c_{\gamma-2} & c_{\gamma-3} & \cdots & c_{0} & -M_{1} \\
\vdots & \vdots & \vdots & \vdots & \vdots \\
-M_{m-\gamma} & -M_{m-\gamma-1} & \cdots & -M_{m-2} & -M_{m-1}
\end{array}\right]\left[\begin{array}{c}
b_{0} \\
e_{1} \\
\vdots \\
\vdots \\
\vdots \\
e_{\gamma-2} \\
\varepsilon_{\gamma-1}
\end{array}\right]=\left[\begin{array}{c}
c_{t-\gamma-1} \\
-c_{t-\gamma-2} \\
\vdots \\
M_{1} \\
M_{2} \\
\vdots \\
M_{m}
\end{array}\right]
$$

(or) $\mathrm{Pe}=\mathrm{q}$ in matrix vector form

Eq. (8) is equivalent to equating all the significant time moments $\left(c_{i}\right)$ and markov parameters $\left(M_{j}\right)$ of $G_{n}(s)$. Where $i=0,1, \cdots, t-1$ and $j=1,2, \cdots, m$.

Step 3: Calculation of ' $e$ ' from this non square system of equation(8) can only done in the least square sense, i.e.:

$$
e=\left[P^{T} P\right]^{-1} P^{T} q
$$


Step 4:Finally the reduced denominator is obtained as:

$D_{r}(s)=e_{0}+e_{1} s+\cdots+e_{r-1} s^{r-1}+s^{r}$

Step 5: Hence from $\operatorname{Eq}(5)$ the reduced numerator polynomial is obtained as:

$N_{r}(s)=d_{0}+d_{1} s+\cdots+d_{r-1} s^{r-1}$

\section{Relative Mapping errors:}

The relative mapping errors of the original model relative to its Reduced model are expressed by means of the relative integral square error criterion, which are given by [11] :

$I=\int_{0}^{\infty}\left[H(t)-H_{r}(t)\right]^{2} \cdot d t / \int_{0}^{\infty} H^{2}(t) \cdot d t$

-.(12) Step-4: After obtaining the reduced denominator

$=\int_{0}^{\infty}\left[G(t)-G_{r}(t)\right]^{2} \cdot d t / \int_{0}^{\infty}[G(t)-G(\infty)]^{2} \cdot d t$

... (13) Eq.(5).

Where, $H(t)$ and $G(t)$ are the impulse and step responses of original system, respectively, and $H_{r}(t)$ and $G_{r}(t)$ are that of their approximants.

\section{RESULTS}

Two numerical examples are chosen from the literature to show the flexibility and effectiveness of the proposed reduction algorithm than other existing methods, and the response of the original and reduced models are compared.

Example-1: Letus consider the system described by the transfer function [12]:

$$
G_{4}(s)=\frac{14 s^{3}+248 s^{2}+900 s+1200}{s^{4}+18 s^{3}+102 s^{2}+180 s+120} \cdots(14)
$$

For which a second order reduced model $R_{2}(s)$ is desired.

Step-1: Expand $G_{4}(s)$ about $\mathrm{s}=0$, gives the time moment proportional's $\left(C_{i}\right)$ where $\mathrm{i}=0,1,2 \ldots$ which are shown below.

$G_{4}(s)=10-7.5 s+4.81667 s^{2}-2.2333 s^{3}+\cdots$

Similarly, expand $G_{4}(s)$ about $\mathrm{s}=\infty$ gives the markov parameters $\left(M_{j}\right)$ where $j=1,2,3 \ldots \ldots$ which are shown below

$$
G_{4}(s)=14 s^{-1}-4 s^{-2}-456 s^{-3}-7296 s^{-4}+\cdots \cdots
$$
the numerator coefficients are determined by matching the first (r-1) time moments of the system to the reduced model via the first $(r-1)$ of

Step-2: Taking $t=4$ time moment proportional's and $\mathrm{m}=0$ markov parameters of $G_{4}(s)$ in Eq. (8) gives the reduced denominator coefficients as the solution of:

$$
\begin{gathered}
{\left[\begin{array}{ll}
C_{3} & C_{2} \\
C_{2} & C_{1}
\end{array}\right]\left[\begin{array}{l}
e_{0} \\
e_{1}
\end{array}\right]=\left[\begin{array}{l}
-C_{1} \\
-C_{0}
\end{array}\right]} \\
\text { i.e. }\left[\begin{array}{cc}
-2.2333 & 4.81667 \\
4.81667 & -7.5
\end{array}\right]\left[\begin{array}{l}
e_{0} \\
e_{1}
\end{array}\right]=\left[\begin{array}{c}
7.5 \\
-10
\end{array}\right]
\end{gathered}
$$

Step-3:The reduced denominator coefficients from Eq.(18) are obtained using Eq.(9):Therefore,

$$
D_{r}(s)=s^{2}+2.138151 s+1.253176
$$

Therefore finally $2^{\text {nd }}$ order reduced model is obtained as:

$$
R_{2}(s)=\frac{11.982691 s+12.531758}{s^{2}+2.138151 s+1.253176} \cdots
$$

The proposed method produces quite different reduced models gives the results as shown in Table $\mathrm{I}$, where' $\mathrm{t}$ ' time moments and ' $\mathrm{m}$ ' markov parameters are used to calculate the denominator and the numerator is determined by matching the first $(\mathrm{r}-1)$ time moments of the original system.

\section{TABLE I}

COMPARISION OF SECOND ORDER MODELS BY PROPOSED

\begin{tabular}{|c|c|c|c|c|c|c|c|}
\hline $\mathrm{t}$ & $\mathrm{m}$ & $d_{0}$ & $d_{1}$ & $e_{0}$ & $e_{1}$ & $\mathrm{I}(\%)$ & $\mathrm{J}(\%)$ \\
\hline 4 & 0 & 12.5317 & 11.9826 & 1.2531 & 2.1381 & 1.338 & 0.12 \\
\hline 4 & 1 & 3.90636 & 13.7175 & 0.3906 & 1.6647 & 1.148 & 0.52 \\
\hline 5 & 1 & 8.55231 & 13.4671 & 0.8552 & 1.9881 & 1.046 & 0.39 \\
\hline 6 & 1 & 10.8898 & 13.2789 & 1.0889 & 2.1446 & 1.016 & 0.33 \\
\hline 7 & 1 & 11.9266 & 13.1593 & 1.1926 & 2.2104 & 1.012 & 0.29 \\
\hline
\end{tabular}

A comparison of the proposed algorithm with the other well known existing order reduction techniques for a second-order reduced model, is given in Table II and the values of I and $\mathrm{J}$ are comparable for the proposed and the other existing techniques. The step, impulse and frequency responses of $G_{4}(s)$ and $R_{2}(s)$ are shown in Fig 4(a)-(c), respectively. 
TABLE II

COMPARISON OF REDUCED ORDER MODELS

\begin{tabular}{|c|c|c|c|}
\hline $\begin{array}{l}\text { Method of } \\
\text { Reduction }\end{array}$ & Reduced Models & $\mathrm{I}(\%)$ & $\mathrm{J}(\%)$ \\
\hline $\begin{array}{l}\text { Proposed method } \\
(\mathrm{t}=4 ; \mathrm{m}=0)\end{array}$ & $\frac{11.982691 s+12.531758}{s^{2}+2.13815 s+1.25317}$ & 1.3383 & 0.1275 \\
\hline $\begin{array}{l}\text { GLSM }[8] \\
(\mathrm{t}=3 ; \mathrm{m}=1)\end{array}$ & $\frac{14 s-129.89689}{s^{2}-8.342267 s-12.98969}$ & Unstable & Unstable \\
\hline $\begin{array}{l}\text { GLSM[8] } \\
(\mathrm{t}=4 ; \mathrm{m}=1)\end{array}$ & $\frac{12.039804 s-59.859657}{s^{2}-3.285494 s-5.985966}$ & Unstable & Unstable \\
\hline $\begin{array}{l}\text { GLSM }[8] \\
(\mathrm{t}=5 ; \mathrm{m}=1)\end{array}$ & $\frac{10.89448 s-38.611107}{s^{2}-1.806385 s-3.8611}$ & Unstable & Unstable \\
\hline PSO[13] & $\frac{12.0166 s+12.0226}{1.016 s^{2}+2.1155 s+1.2022}$ & 1.3881 & 0.1143 \\
\hline Gutman et.al[14] & $\frac{17.64706 s+70.58824}{s^{2}+5.2491 s+7.05582}$ & 10.828 & 8.8077 \\
\hline $\begin{array}{c}\text { Shamash } \\
\text { [12] }\end{array}$ & $\frac{8.83 s+11.76}{s^{2}+1.765 s+1.176}$ & 6.0030 & 1.4698 \\
\hline Chen et al[15] & $\frac{8.8927 s+11.9036}{s^{2}+1.78554 s+1.19036}$ & 5.8277 & 1.3818 \\
\hline Lucas[16] & $\frac{30 s+40}{3 s^{2}+6 s+4}$ & 3.5426 & 0.5256 \\
\hline
\end{tabular}

\begin{tabular}{|c|c|c|c|}
\hline Original Order system & $\begin{array}{l}\text { Interpola } \\
\text { tion } \\
\text { points }\end{array}$ & $\begin{array}{l}\text { Reduced order } \\
\text { system }\end{array}$ & ISE \\
\hline$G(s)=\frac{s^{4}+13 s^{3}+63 s^{2}+133 s+102}{s^{6}+14.5 s^{5}+81 s^{4}+223 s^{3}+318 s^{2}+21}$ & $\begin{array}{l}0,-0.5,0 \\
9+1.78 \mathrm{i} \\
0.9-1.78 \mathrm{i}\end{array}$ & $R_{2}(s)=\frac{1.34 s+0}{s^{2}+0.75838}$ & 0.186 \\
\hline$G(s)=\frac{s^{4}+13 s^{3}+63 s^{2}+133}{s^{6}+14.5 s^{5}+81 s^{4}+223 s^{3}+318}$ & $0,0.6,2,3$ & $R_{2}(s)=\frac{0.0809 s}{s^{2}+0.75838}$ & 0.148 \\
\hline$G(s)=\frac{s^{4}+13 s^{3}+63 s^{2}+133}{s^{6}+14.5 s^{5}+81 s^{4}+223 s^{3}+318}$ & $\begin{array}{l}0,-3.528 \\
9, \\
0.11+1.7 \\
8 \mathrm{i}, \\
0.11-1.7 \\
8 \mathrm{i}\end{array}$ & $R_{2}(s)=\frac{0.261988 s}{s^{2}+0.75838}$ & $\begin{array}{l}0.042 \\
34\end{array}$ \\
\hline
\end{tabular}

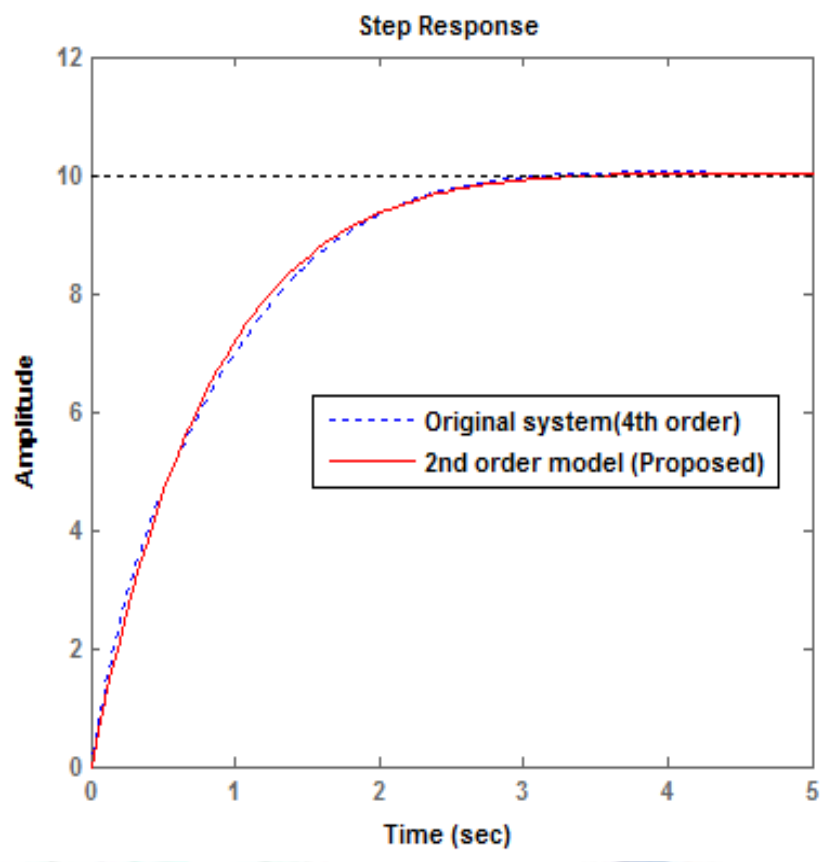

Fig. 4 Comparison of step responses of $G_{4}(s)$ and $R_{2}(s)$

Example 2: Consider the $8^{\text {th }}$ order system as follows:

$$
\begin{gathered}
\mathrm{G}(\mathrm{s})=\frac{d_{n}(s)}{e_{n}(s)} \\
d_{n}(s)=0.01141 s^{7}+0.5119 s^{6}+2.4152 s^{5}+4.918 s^{4} \\
+6.2164 s^{3}+4.6146 s^{2} \\
+1.7134 s+0.261 \\
e_{n}(s)=s^{8}+9.83 s^{7}+36.616 s^{6}+65.852 s^{5}+73.018 s^{4} \\
+50.03 s^{3}+17.104 s^{2} \\
+1.919 s+0.25
\end{gathered}
$$

A Second order reduced model is obtained for the above higher order system, in following steps, using the proposed method given in section-3.

$R_{2}(s)=\frac{a_{0}+a_{1} s}{b_{0}+b_{1} s+b_{2} s^{2}}=\frac{a_{k}(s)}{b_{k}(s)}$

By normalizing the above, the reduced order denominator is(by applying routh method):

$b_{k}(s)=s^{2}+0.07881 s+0 . .0186$

Reduced order numerator (by applying Interpolation method):

Using the procedure the optimum reduced order model is obtained as,

$$
R_{2}(s)=\frac{0.05244 s+0.0194}{s^{2}+0.07881 s+0.0186}
$$




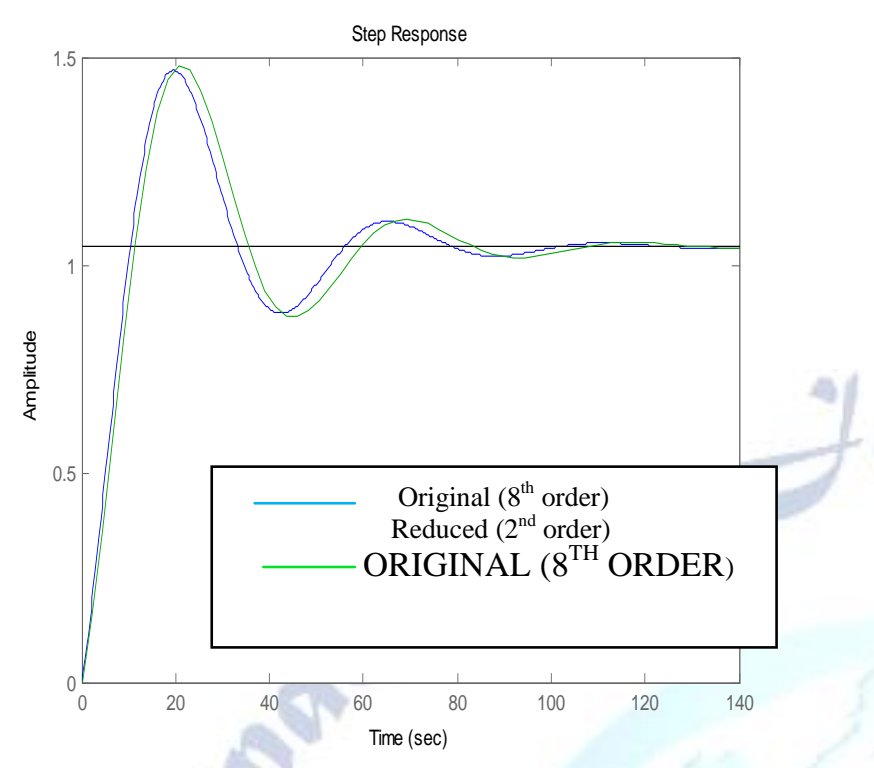

Fig 2 Step response of original and reduced order systems.

Design of PID controller for reduced order model is, Let transfer function of the PID controller is,

$$
G_{c}(s)=\frac{k_{d} s^{2}+k_{p} s+k_{i}}{s}
$$

Applying ITAE performance index method to the reduced model, the values of $\mathrm{Kp}, \mathrm{Ki}$ and $\mathrm{Kd}$ are obtained

$$
R_{2}(s)=\frac{0.05244 s+0.0194}{s^{2}+0.07881 s+0.0186}
$$

Now to obtain the closed-loop transfer function for reduced order

The tuned PID values are

$$
K_{p}=-9.1331, \quad K_{i}=7.654, \quad K_{d}=23.899
$$

Comparing characteristic equation of compensated system to the optimum ITAE characteristic equation as,

$$
s^{3}+1.75 w_{n} s^{2}+3.25 w_{n}^{2} s+w_{n}^{3}
$$

The PID controller is added to the forward path and the closed loop transfer function with unity feedback of the system is given as:

$$
T_{c}(s)=\frac{G_{C}(S) G(S)}{1+G_{C}(S) G(S)}
$$

Where $G(s)$ is the high order system and $G_{c}(s)$ is the PID controller transfer function.

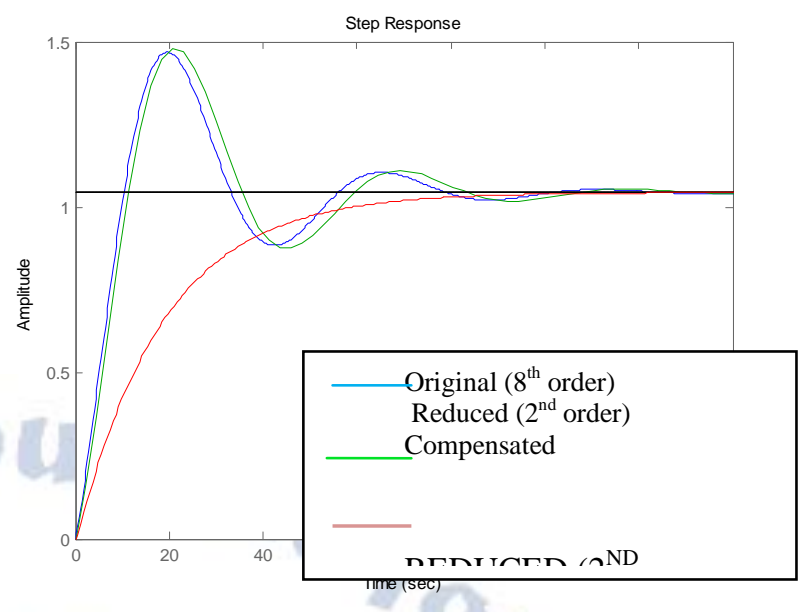

Fig 3 Step response of compensated system and uncompensated systems

\section{IV.DISCUSSION}

The concept of order reduction by generalized least squares method has been improved and employed to determine the reduced denominator polynomials and numerator polynomials are obtained by matching initial time moments of the original model. The proposed method generates better approximations of a higher order linear, time invariant dynamic systems. The relative step and impulse mapping errors between the original and reduced order models are also determined and plotted with respect to time. A comparison of these mapping errors for the proposed reduction method and the other well-known existing order reduction techniques is also given, from which it is clear that the proposed method compares well with the other existing techniques. The results show that the proposed method leads to good and stable reduced models for linear time invariant systems.

\section{REFERENCES}

[1] R. Genesio and M. Milanese, "A note on the derivation and use of reduced order models", IEEE Trans. Automat.Control,Vol. AC-21,No. 1, pp. 118-122, February 1976.

[2] M.Jamshidi, Large Scale Systems Modelling and Control Series, NewYork, Amsterdam, Oxford, North Holland, Vol. $9,1983$.

[3] C.S.Hsien, C.Hwang, "Model Reduction of Continous time Systems using a modified Routh Approximation Method" IEE Proceedings, Vol.136, Pt.d, No.4, July-1989.

[4] Y.Shamash, "Approximations of linear time invariant systems", Proc.Conf. on Pade approximants and their Applications, P. R. Graves-Morris(Ed.), London Academic, 1973.

[5] A. Bultheel and M. V. Barel, "Pade techniques for model reduction in linear system theory, A survey", Journal of Computational and Applied Mathematics, Vol. 14, pp.401-438, 1986 . 
[6] F. F. Shoji, K. Abe and H. Takeda, "Model reduction for a class of linear dynamic systems", Journal of Franklin Inst., Vol.319, pp. 549-558, 1985.

[7] T. N. Lucas and I. F. Beat,"Model reduction by least squares moment matching", Electronics Letters, Vol. 26, No. 15, pp. 1213-1215, July 1990.

[8] T. N. Lucas and A. R. Munro, "Model reduction by generalisedleast squares method", Electronics Letters, Vol. 27, No. 15, pp. 1383-1384, July 1991.

[9] L. A. Aguirre, "The least-squares Padé method for model reduction", Int Journal of Systems Science, Vol. 23, No. 10, pp. 1559-1570, 1992.

[10] G.Parmar, R.Prasad and S.Mukherjee, "Order Reduction by Least-Squares Methods about General point 'a"' , world Academy of Science, Engineering and Technology 262007.

[11] T.N. Lucas, " Further discussion on impulse energy Approximation " IEEE Trans. Automat. Control, Vol. AC-32, No. 2, pp. 189-190,February 1987.

[12] Y. Shamash, "Model reduction using the Routh stability criterion andthe Pade approximation technique", Int. J. Control, Vol. 21, pp 475-484,1975.

[13] S.Panda, S.K.Tomar, R.Prasad, C.Ardil, "Reduction of Linear Time-Invariant Systems Using Routh-Approximation and PSO", International Journal of Applied Mathematics and Computer Science 5:2 2009.

[14] P. O. Gutman, C. F. Mannerfelt and P. Molander, "Contributions to themodel reduction problem", IEEE Trans. Auto. Control, Vol. 27, pp 454-455, 1982.

[15] T. C. Chen, C. Y. Chang and K. W. Han, "Reduction of transferfunctions by the stability equation method", Journal of FranklinInstitute, Vol. 308, pp 389-404, 1979.

[16] Lucas T N, IEE Proc, Pt.D, 130(6) (1983) 362-364.

[17] T.N.Lucas, "Linear system reduction by the modified factor division method" , IEEE Proceedings Vol.133 Part D No.6, Nov-1986, pp293-295.

[18] J.S.Yadav, N.P.Patidar, J.Singhai, S.Panda and C.Ardil, "A Combined conventional and differential evolution method for model order reduction", International Journal of Compuational Intelligence 5:2 2009. 